\title{
Development of a constitutive law for numeri- cal simulation of artificial leaflet-structures for transcatheter heart valve prostheses
}

\begin{abstract}
While the current generation of devices for minimally invasive treatment of severe symptomatic aortic valve stenosis is based on xenogenic leaflet-material, artificial polymeric leaflet-structures represent a promising approach for future improvement of heart valve performance. For enhanced long-term success of polymeric leafletstructures, limitations regarding calcification and durability have to be addressed. The objective of the presented study was the development of a constitutive law representing the material properties of artificial polymeric leaflet-structures of transcatheter heart valve prostheses in numerical simulation to assess the in silico leaflet-performance. Mechanical characterization of cast films and nonwoven specimens of a polycarbonate based silicone elastomer were conducted by means of uniaxial tension and planar shear testing, respectively. For validation purposes, experimental data were compared with the results of finite-element analysis (FEA) using different hyperelastic models. Strain energy function for third-order ogden hyperelastic model achieved the best fit of the non-linear stress-strain behavior of the isotropic polymeric material with the experimental data. It was chosen for further FEA of valve leaflet-performance under physiological pressurization to analyze the suitability of various manufacturing processes for polymeric leafletstructures. Therefore a specific leaflet-design with a wall thickness of $400 \mu \mathrm{m}$ was used. As a result of FEA, time dependent leaflet-deformation, leaflet coaptation surface area (CSA) and leaflet opening area (LOA) of cast and nonwoven leaflet-structures were calculated. While LOA was comparable for cast and nonwoven leaflet-structures,
\end{abstract}

\footnotetext{
*Corresponding author: Sylvia Pfensig: Institute for ImplantTechnology and Biomaterials e.V., Friedrich-Barnewitz-Str. 4, 18119 Rostock-Warnemünde, Germany,

e-mail: sylvia.pfensig@uni-rostock.de

Daniela Arbeiter, Stefanie Kohse, Niels Grabow, Klaus-Peter Schmitz: Institute for Biomedical Engineering, Rostock University Medical Center, 18119 Rostock-Warnemünde, Germany

Sebastian Kaule, Michael Stiehm, Klaus-Peter Schmitz, Stefan Siewert: Institute for ImplantTechnology and Biomaterials e.V., 18119 Rostock-Warnemünde, Germany
}

obtained leaflet-dynamics in a cardiac cycle under physiological pressurization demonstrated crucial influence of the manufacturing process. For nonwoven leafletstructures, an enhanced CSA could be demonstrated in comparison to cast structures. FEA using a validated hyperelastic constitutive law represents a useful tool for in silico performance evaluation of polymeric leaflet-structures under physiological loading and proves the suitability of the polymeric artificial leaflet-material for percutaneous heart valve prostheses.

Keywords: Finite-element analysis, transcatheter heart valve prosthesis, polymeric leaflet-structures, constitutive law.

https://doi.org/10.1515/cdbme-2019-0143

\section{Introduction}

The future generation of devices for minimally invasive treatment of aortic valve stenosis will benefit from leafletstructures based on artificial polymeric materials. Therefore, crucial improvenents concerning heart valve performance, durability, calcification and shelf life properties represent key aspects of the current research in the field of polymeric leaflet-structures [1,2].

Finite-element analysis (FEA) represents an essential tool for the design development of heart valve leafletstructures. The mechanical behavior and in silico deformation of aortic heart valve leaflets under physiological loading and the influence of various design parameters on its performance has been investigated in structural mechanical FEA, previously [3-5]. Within the current work, we developed and validated a hyperelastic constitutive law for polymeric leaflet-structures for FEA. The influence of the constitutive law on time-dependent leaflet-deformation within a physiological cardiac cycle was analyzed with regard to leaflet coaptation surface area and leaflet opening area. Furthermore, the suitability of various manufacturing processes for polymeric artificial leaflet-material for percutaneous heart valve prostheses was assessed. 


\section{Materials and methods}

\subsection{Design of artificial leaflet-structure}

The leaflet-structure was designed as axially-symmetric trileaflet heart valve geometry in an unpressurized and closed configuration as a stress-free state. For modelling, the computer-aided design (CAD) software Creo Parametric 5.0 (Parametric Technology Corp., Needham, MA, USA) was used, taken previously published literature on leafletgeometry into account for design development [5].

A single leaflet was designed using the parameters summarized in Table 1 and patterned three times, rotated by $120^{\circ}$ around the centre of the valve. The model is basically built by the valve height and base diameter. A leaflet wall thickness of $400 \mu \mathrm{m}$ was used. For the lunula angle, a value of 34 degrees was chosen in accordance with further design studies [5]. The belly line is formed by the three parameters commissure angle, coaptation angle and curvature of the belly. Figure 1 illustrates the described design parameters of the trileaflet heart valve model.

Table 1: Design parameters and corresponding values describing the leaflet-design in unpressurized and closed configuration.

\begin{tabular}{lll}
\hline Symbol & Design parameter & Value \\
\hline$H_{V}$ & Valve height & $18.5 \mathrm{~mm}$ \\
$D_{b}$ & Base diameter & $26.0 \mathrm{~mm}$ \\
$s$ & Leaflet-thickness & $400 \mu \mathrm{m}$ \\
$\Omega$ & Lunula angle & $34^{\circ}$ \\
$\varphi$ & Symmetry angle & $120^{\circ}$ \\
$R_{A}$ & Arc radius & $2.0 \mathrm{~mm}$ \\
$\alpha$ & Commissure angle & $20^{\circ}$ \\
$\beta$ & Coaptation angle & $80^{\circ}$ \\
$C$ & Curvature of the belly & 0.5 \\
\hline
\end{tabular}

A)

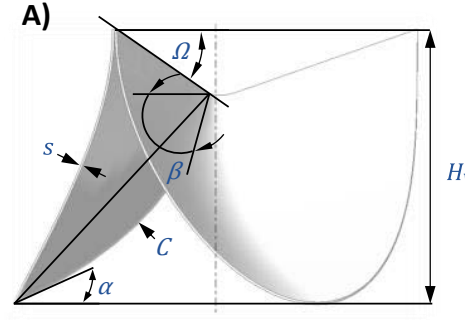

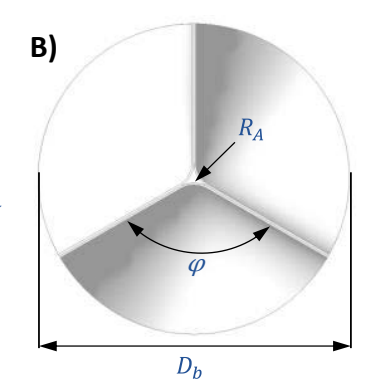

Figure 1: Side (A) and Top view (B) of the heart valve model: $H_{V}$ valve height, $\Omega$ lunula angle, $\alpha$ commissure angle, $\beta$ coaptation angle, $C$ curvature of the belly, $s$ leaflet-thickness, $D_{b}$ base diameter, $\varphi$ symmetry angle and $R_{A}$ arc radius.

\subsection{Development of a constitutive law}

As artificial leaflet-material for polymeric leaflet-structures a polycarbonate based silicone elastomer was chosen. For the development of a hyperelastic constitutive law for the artificial leaflet-structures, cast films and nonwoven specimens were manufactured by means of dip coating and electrospinning, respectively.

Mechanical characterization was conducted by means of uniaxial tension and planar shear testing. Cast films $(n=10)$ were analyzed at room temperature, because no influence of a saline solution was observed. Nonwoven specimens ( $n=10)$ were analyzed in $0.9 \%$ saline solution at $37^{\circ} \mathrm{C}$, using a universal testing machine Zwick/Roell Z2.5/TN (Zwick GmbH \& Co. KG, Ulm, Germany).

Figure 2 presents the dimensions of rectangular specimens and illustrates the stress-strain behavior of cast films and nonwoven specimens during uniaxial tension and planar shear testing.

A)

uniaxial tension (UT)

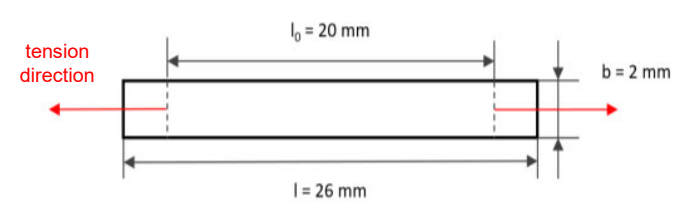

B)
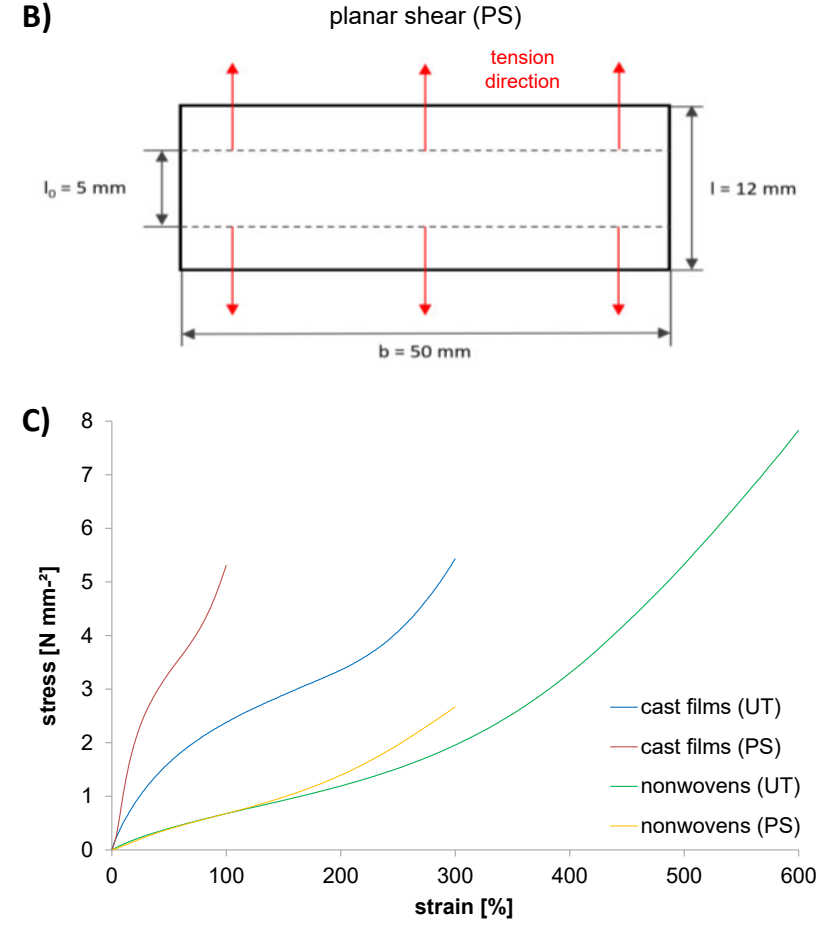

Figure 2: Dimensions of specimens for uniaxial tension (A) and planar shear testing (B) and averaged curves of the stress-strain behavior of mechanically characterized cast films and nonwoven specimens by means of uniaxial tension and planar shear testing (C). 


\subsection{Finite-element analysis}

For validation purposes, experimental determined data of uniaxial tension and planar shear testing were compared with numerical results of FEA using ABAQUS/Standard 2017 (Dassault Systèmes, Vélizy-Villacoublay, France). Numerical stress-strain behavior for different hyperelastic models of order $N$ was evaluated with regard to stability and accuracy compared to the test data for cast films and nonwoven specimens, respectively. The strain-energy models of Ogden, Mooney-Rivlin and Neo-Hookean were used for validation.

Furthermore FEA (ABAQUS/Explicit 2017, Dassault Systèmes, Vélizy-Villacoublay, France) was used for numerical simulation of the leaflet-dynamics during two complete cardiac cycles with a duration of $0.854 \mathrm{~s}$, respectively. In vitro hydrodynamic testing of a commercially available heart valve prosthesis was used in order to define physiological ventricular and aortal pressure conditions for numerical simulations [5]

For fixation, the displacement of the commissure was constrained. Surface-to-surface contact was applied between neighboured leaflets using a friction coefficient of 0.3 .

A generalized isotropic hyperelastic constitutive law with a density of $10^{-9} \mathrm{~kg} / \mathrm{mm}^{3}$ and a Poisson's ratio of 0.475 was defined to describe the polymeric material properties. Meshing was conducted, using a total of 9,474 C3D8 continuum elements with a global edge length of $0.40 \mathrm{~mm}$.
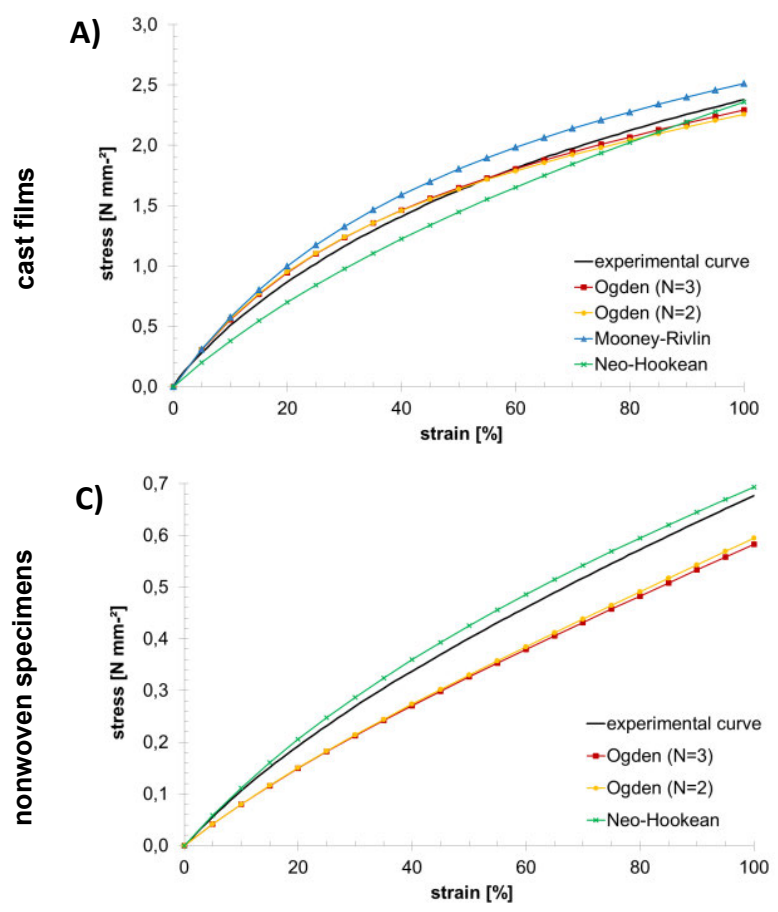

\section{Results and discussion}

\subsection{Material model for polymeric leaflets}

Strain energy function for third-order Ogden $(N=3)$ hyperelastic model achieved the best fit of the experimental data and full stability of the non-linear stress-strain behavior of the isotropic polymeric material (see Figure 3). It was chosen for further FEA of valve leaflet-performance under physiological pressurization to analyze the in silico deformation and assess the suitability of cast and nonwoven leaflet-structures for future heart valves (see Table 2).

Table 2: Material parameters of stable third-order Ogden hyperelastic model for cast films ( $1^{\text {st }}$ line $)$ and nonwovens $\left(2^{\text {nd }}\right.$ line $)$.

\begin{tabular}{cccc}
\hline $\mathrm{I}$ & $\boldsymbol{\mu}_{\mathrm{i}}$ & $\boldsymbol{\alpha}_{\mathrm{i}}$ & $\mathrm{D}_{\mathrm{i}}$ \\
\hline $\mathbf{1}$ & 0.2101 & 2.8384 & $\begin{array}{c}4.5010 \mathrm{E}-02 \\
0.3498\end{array}$ \\
\hline $\mathbf{2}$ & 0.2892 & 2.5195 & 0 \\
\hline $\mathbf{3}$ & $1.1649 \mathrm{E}-07$ & 13.9474 & \\
& $2.0215 \mathrm{E}-04$ & 6.6970 & 0 \\
\hline
\end{tabular}
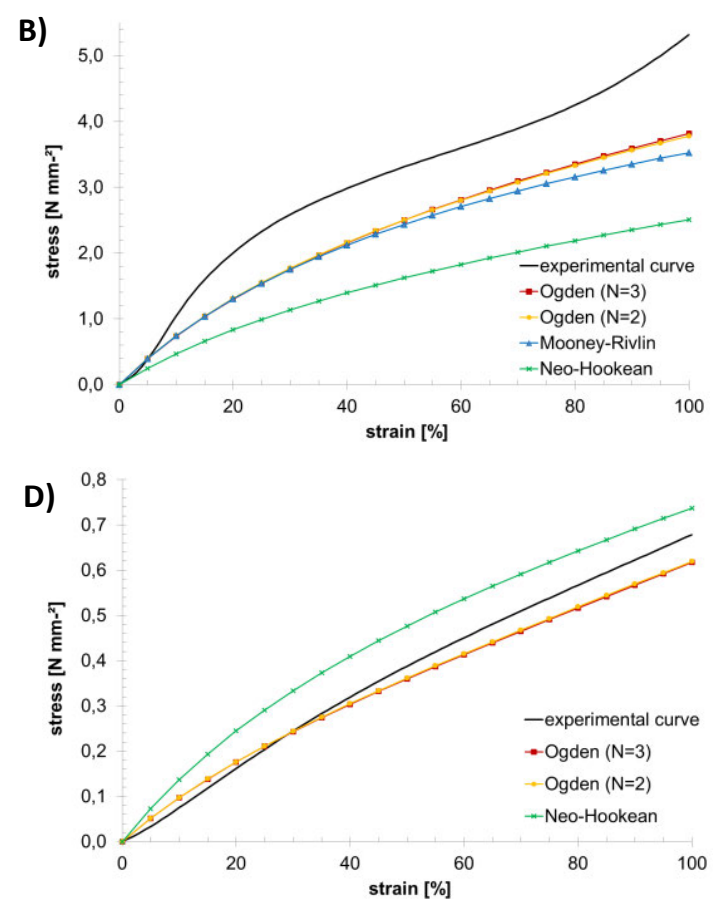

Figure 3: Comparison of stress-strain behavior of an averaged curve based on experimental investigations and numerical results of FEA using different hyperelastic models: uniaxial tension $(\mathbf{A}, \mathbf{C})$ and planar shear $(\mathbf{B}, \mathbf{D})$ of cast films and nonwoven specimens. 


\subsection{Leaflet-dynamics in a cardiac cycle}

As a result of FEA, time dependent leaflet-deformation, leaflet coaptation surface area (CSA) and leaflet opening area (LOA) of cast and nonwoven leaflet-structures was calculated (see Figure 4). While the maximum LOA within a cardiac cycle was comparable for cast $\left(3.78 \mathrm{~cm}^{2}\right)$ and nonwoven $\left(3.93 \mathrm{~cm}^{2}\right)$ leaflets, obtained leaflet-dynamics under physiological pressurization demonstrated crucial influence of the manufacturing process of the polymeric leafletstructures. The duration of systole for cast leaflets was less than half the time for nonwovens. Furthermore, an enhanced CSA could be demonstrated for nonwoven $\left(37 \mathrm{~mm}^{2}\right)$ in comparison to cast leaflet-structures $\left(79 \mathrm{~mm}^{2}\right)$. The current findings indicate better suitability of the polymeric nonwoven leaflet-material compared to the cast leaflet-material.
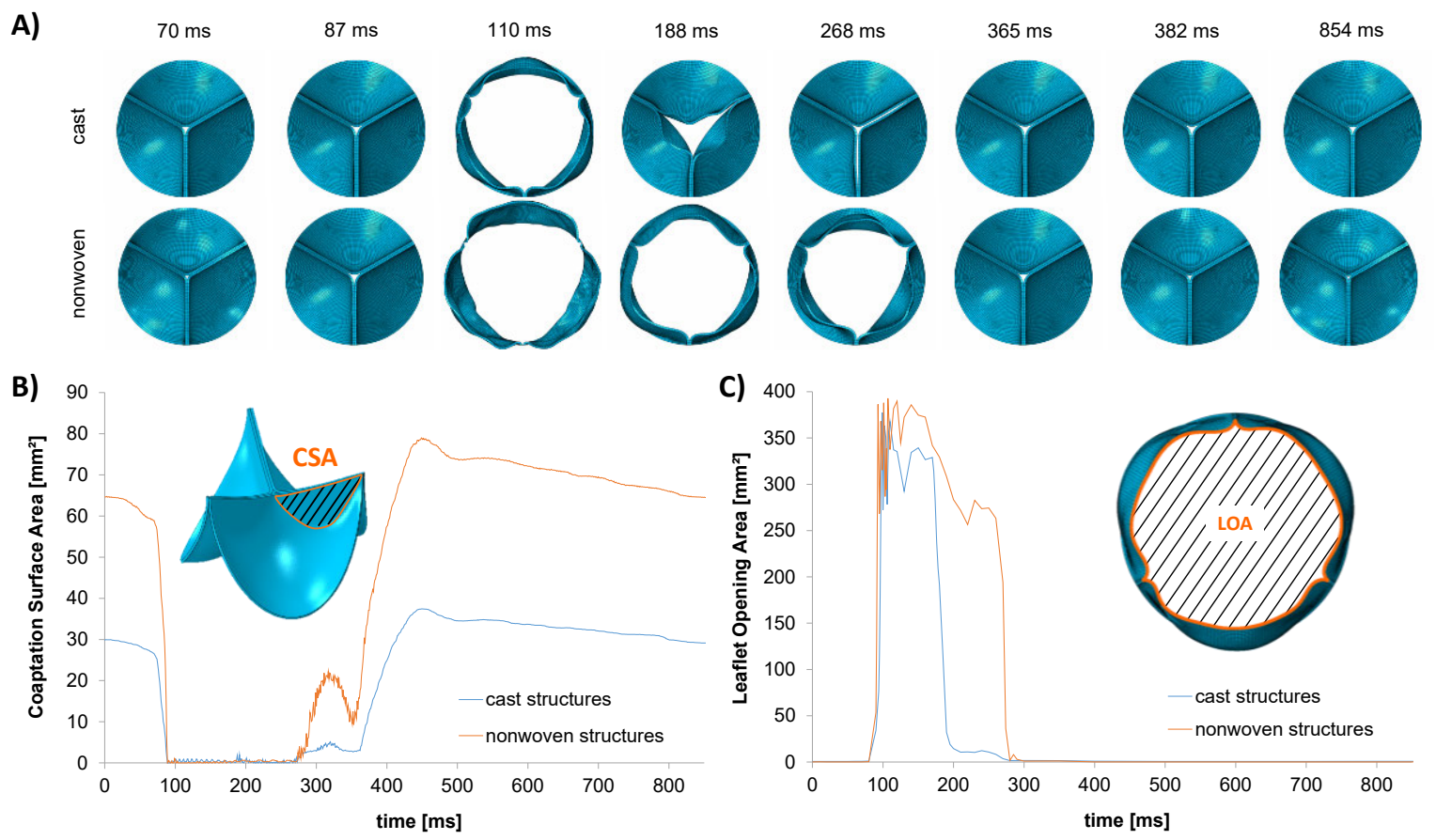

Figure 4: Results of FEA: performance of cast and nonwoven leaflet-structures according to aortic and ventricular pressure curves (A), average coaptation surface area (B) and leaflet opening area (C) during opening and closing for a cardiac cycle of 0.854 s.

\section{Conclusion}

Within the current study, we developed a hyperelastic constitutive law for a polycarbonate based silicone elastomer describing the non-linear stress-strain behaviour of the isotropic artificial leaflet-material. FEA represents a useful tool for in silico performance evaluation of polymeric cast and nonwoven leaflet-structures under physiological loading and demonstrated the better suitability of the nonwoven leafletmaterial for future percutaneous heart valve prostheses.

\section{Author Statement}

Research funding: Financial support by the European Regional Development Fund (ERDF) and the European Social Fund (ESF) within the collaborative research between economy and science of the state Mecklenburg-Vorpommern within the project "TheraVista" is gratefully acknowledged. Conflict of interest: Authors state no conflict of interest.

\section{References}

[1] Claiborne TE, Slepian MJ, Hossainy S, Bluestein D. Polymeric trileaflet prosthetic heart valves: evolution and path to clinical reality. Expert Rec Med Devices 2012;9(6):577-594.

[2] Bezuidenhout D, Williams DF, Zilla P. Polymeric heart valves for surgical implantation, catheter-based technologies and heart assist devices. Biomaterials 2016;36:6-25.

[3] Labrosse MR, Lobo K, Beller CJ. Structural analysis of the natural aortic valve in dynamics: From unpressurized to physiologically loaded. Journal of Biomechanics 2010;43:19161922.

[4] De Gaetano F, Bagnoli P, Zaffaro A, Pandolfi A, Serrani M, Brubert J, Stasiak J, Moggridge GD, Constantino ML. A Newly Developed Tri-Leaflet Polymeric Heart Valve Prosthesis. J Mech Med Biol. 2015;15(2).

[5] Pfensig S, Kaule S, Sämann M, Stiehm, M, Grabow N, Schmitz KP, Siewert S. Assessment of heart valve performance by finite-element design studies of polymeric leafletstructures. Current Directions in biomedical Engineering 2017;3(2):631-634. 